\title{
Adapting Best Path for Mobile Robot By Predicting Obstacle Size
}

\author{
Ramesh Dadi ,Sallauddin MD, Syed Nawaz Pasha, A. Harshavardhan, P.Kumarawamy
}

\begin{abstract}
In this paper we are proposing an approach to find obstacle size to reduce the searching area for path planning for mobile robot. With this approach, the vehicle is able to reduce searching area compare to other algorithms. This is main problem normally all the algorithms were concentrate how to optimal path but without reducing searching area it's not possible. The approach we use in our model consists of various kinds of obstacles such as motor cycles, pedestrians, animals, etc. The presence of and the appropriate path is planned according to the dimensions of the obstacle as different obstacles have different dimensions.
\end{abstract}

Keywords: Optimal path, varying obstacle, dynamic

\section{I.INTRODUCTION}

One of the major issues in path planning in autonomous vehicles is to plan the ideal way from source to goal in static and changing environment. All the path planning algorithms play an important role in estimating the accurate path. These algorithms try to find the optimal path by moving from one place to other place in any environment considering the starting and ending points. To accomplish this number of sensors are incorporated that sense the obstacle and move through different coordinates given the source and destination. So our approach of path planning in driverless car is to optimize the local path by sensing the obstacle size as if we predict the accurate obstacle size then the searching area will also be reduced.

\section{II.RELATED WORK}

One of the major issues paths planning for mobile robots is to search out the best path from source to goal in constant and changing environments. So the way discovering obstacle free path is important in a changing environment. Way arranging calculations have the obligation of finding ideal way starting with one spot then onto the next spot in any condition by taking a beginning stage and a goal point for this, an adjunct of sensors are playing major role in finding the path to move the vehicle. These sensors are responsible for choosing the appropriate path in changing

Revised Manuscript Received on July 18, 2019.

Ramesh Dadi, Computer Science \& Engineering, S R Engineering College, Warangal, India. Email- dadiramesh44@gmail.com

Sallauddin MD, Computer Science \& Engineering, S R Engineering College, Warangal, India

Syed Nawaz Pasha, Computer Science \& Engineering, S R Engineering College, Warangal, India

A. Harshavardhan, Computer Science \& Engineering, S R Engineering College, Warangal, India

Nirzari Pa Computer Science \& Engineering, S R Engineering College, Warangal, India

P.Kumarawamy, Computer Science \& Engineering, S R Engineering College, Warangal, India environment.

environment [4] [9] when we give start and end positions. So psychological based way arranging calculation finds a way with utilizing subjective science and versatile based approaches like how an individual carries on when he was in obscure condition. On this unmistakable the versatile design of the Cro-Magnon man is executed as individuals add up to be asked debut where one is going when they have ensure to integral data.

There are distinctive kind of calculations are there to handle this issue of finding the best way from source to goal. The way arranging must be finished remembering the last goal. The viewpoint arranging is light as a quill if there are no deterrents, not withstanding if there is any difficulty in the direction from source to goal before we need to see for perfect ways. A home of that a way organizing estimations have been realized.

\subsection{Dijkstra's algorithm}

Dijkstra's [3][5](named after its engineer, E. Dijkstra ) calculation takes a gander at the neighbors of the hub nearest to the begin, and sets or updates their good ways from the begin. The Dijkstra Algorithm broadens the center point that is most remote from the start center point, so it winds up "bumbling" into the objective hub. Much the same as the broadness first hunt; it is ensured to locate the briefest way.

\subsection{A* algorithm :}

A* algorithm[6][7] is a space-search algorithm it finds path from source to destination based on heuristic value. This Algorithm works same like the Dijkstra and best-first Algorithm is not reducing searching area in finding a path just it offers qualities to the hubs in an unexpected way. Every hub's worth is the total of the genuine expense to that hub from the beginning and the heuristic assessment of the rest of the expense from the hub to the objective. But this algorithm finds a path after searching maximum (80\%) area.

\subsection{Cognitive-Based Adaptive Path Planning Algorithms}

The approach of the CBAPPA [1] [2] is mainly works on cognitive-based steps like how human being behaves in unknown environment to reach the goal. Every movement is calculated based different heuristic calculations. Practically, CBAPPA is finds the way into two distinct stages: In the principal organize, it finds a straight line from source to goal; this straight line will turn into the ideal way at any expense for any calculation. In the event that the goal position is obscure, at that point robot attempts to discover the bearing of the goal from source perspective. This 
straight line will turn into the manual for the robot in the subsequent stage. In the subsequent stage, robot begins advancing toward the goal by defining middle of the road objectives by defeating the hindrance until come to the destination and it uses straight line as a guide. In the subsequent stage, it begins pushing toward the goal by defining transitional objectives by beating the deterrent and by utilizing straight line as a guide the robot will consider just the obstruction which truly intrudes on robot movement in tactile territory like the impediments which are fall in the sensor run. At the point when a deterrent is found inside tactile range and it checks whether the obstruction is static or dynamic in the event that it is static hindrance, at that point it frames a half hover around the snag and pursue the half hover until it arrives at the opposite side of the impediment. So the half circle both the finishes are on the straight line. Like that, it defines middle of the road objectives and stays away from the hindrance until it arrives at the last goal. The above path planning algorithms will find optimal path given the source and destination, but it will not try to find out the actual obstacle size which is also a factor of optimal path planning. In the below approach we propose a way of detecting the optimal obstacle size which optimizes the search area.

\section{DISSCUSSION \& RESULTS}

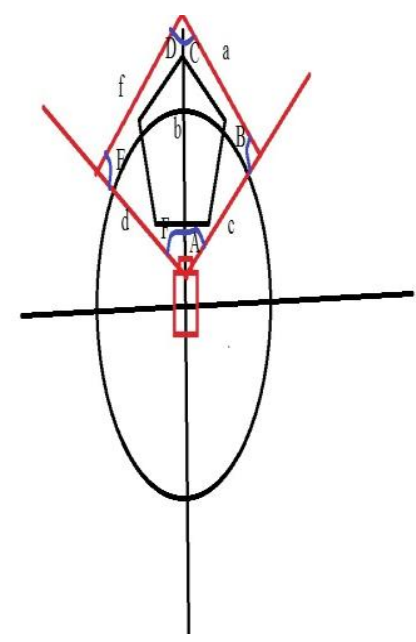

Fig: Path planning in wireless car

The above is the schematic view of the wireless car and a static obstacle with various angles formed to predict the obstacle size.

Let us consider A, B, C, D, E, F are the angles formed by the wireless car.

Let us consider $\mathrm{a}, \mathrm{b}, \mathrm{c}, \mathrm{d}$, e, f are the sides formed using the angular approach.

Approach:

The driverless car will first sense the obstacle and then form the angles relative to the obstacle size. In the given diagram say $\mathrm{A}, \mathrm{F}$ are the angles formed from the given position. It will select the path which formed the least angle. Let $\mathrm{c}$ is the distance travelled by the car after forming the angle. After travelling through some distance again it will sense and form an angle, let B is the angle formed.
From the trigonometric equations:

$\mathrm{a}=\mathrm{BC}, \mathrm{b}=\mathrm{AC}, \mathrm{c}=\mathrm{AB}$

$\mathrm{C}=180-(\mathrm{A}+\mathrm{B})$

$$
\begin{gathered}
b=\frac{a \sin B}{\sin A} \\
\frac{a}{\sin A}=\frac{c}{\sin C} \\
a=\frac{c \sin A}{\sin C} \\
\triangle A B C=\frac{1}{2} a c \sin B
\end{gathered}
$$

From figure angle $\mathrm{B} \equiv E \mathrm{E}$

From figure: $b=D F, f=D E, d=E F$

$$
\begin{gathered}
f=\frac{b \sin F}{\sin E} \\
\Delta D E F=\frac{1}{2} b f \sin D
\end{gathered}
$$

Obstacle size $=\triangle A B C+\triangle D E F E F$

From the above trigonometric calculations we determine the approximate area of the obstacles through triangles formed. Based on the area of the triangle formed the driverless car will opt the path which has less area.

Let us consider that area of $\triangle A B C>\triangle D E F E F$ then it will follow the path through " $\mathrm{d}-\mathrm{f}$ " else it will follow the other path through "a- c".

\section{CONCLUSION:}

At the present time, there is an enormous improvement in path planning which is inspired by new applications in different sectors. There are several techniques available for solving the path planning problem in dynamic environments. In this paper we proposed an approach to find the size of an obstacle and plan an optimal path in any environment which can be used in driverless car. This approach will be used to optimize the search area and improve the efficiency.

\section{REFERENCES}

1. A NOVEL APPROACH TO PATH PLANNING OF ROBOTS BY ETECTING DYNAMIC OBSTACLES. Syed Nawaz Pasha, D. Ramesh \& G Roopa

2. D. W. Payton, J. K. Rosenblatt and D. M. Keirsey, "Gridbased mapping for autonomous mobile robot," Robotics and Autonomous Systems, vol. 11, no. 1, pp. 13-21, 1993.

3. A metaheuristic approach to solve Dynamic Vehicle Routing Problem in continuous search space.by Micha ${ }^{\mathrm{a}}$ Okulewicza,_, Jacek Ma«dziuka

4. A. Stevens and P. Coupe, "Distortions in Judged Spatial Relations," Cognitive Psychology,10(4), 1978, pp. 526550.

5. Hart P. E., Nilsson N. J., Raphael B., A Formal Basis for the Heuristic Determination of Minimum Cost Paths, IEEE Transactions on Systems Science and Cybernetics SSC4 (2):pp. 100-107, 1968.

6. C.-K. Yap, "Algorithmic motion planning," in Advances in Robotics vol. 1: Algorithmic and Geometric Aspects of Robotics, J. T. Schwartz and C.-K. Yap, Eds. Hillsdale, New Jersey: Lawrence Erlbaum, 1987 
7. A. Stentz, "The Focussed D* Algorithm for Real-Time Replanning", Proceedings of International Joint Conference on Artificial Intelligence, 1995, pp. 12131221.

8. Biologically Inspired Visual Odometry Based on the Computational Model of Grid Cells for Mobile Robots Huimin Lu 2016 IEEE.

9. Robotic Motion Planning in Unknown Dynamic Environments: Existing Approaches and Challenges2015 IEEE International Symposium on Robotics and Intelligent Sensors (IEEE IRIS20I5 